\title{
Editorial: Photochromic Materials: Design and Applications
}

\author{
Frederico B. De Sousa ${ }^{1+*}$, Frank Alexis ${ }^{2 \dagger *}$ and Silvia Giordani ${ }^{3+*}$ \\ ${ }^{1}$ Laboratório de Sistemas Poliméricos e Supramoleculares, Instituto de Física e Química, Universidade Federal de Itajubá, Itajubá, \\ Brazil, ${ }^{2}$ School of Biological Sciences and Engineering, Yachay Tech University, Urcuquí, Ecuador, ${ }^{3}$ School of Chemical Sciences, \\ Dublin City University, Glasnevin, Ireland
}

Keywords: photochromic materials, stimuli-responsive material, polymers, light, hybrid materials

Editorial on the Research Topic

Photochromic Materials: Design and Applications

\section{OPEN ACCESS}

Edited and reviewed by: Weihua $L i$,

University of Wollongong, Australia

*Correspondence:

Frederico B. De Sousa

fredbsousa@unifei.edu.br

Frank Alexis

falexis@yachaytech.edu.ec

Silvia Giordan

silvia.giordani@dcu.ie

${ }^{\dagger}$ These authors have contributed equally to this work and share first authorship

Specialty section: This article was submitted to Smart Materials, a section of the journal

Frontiers in Materials

Received: 03 June 2021 Accepted: 08 June 2021 Published: 29 July 2021

Citation:

De Sousa FB, Alexis F and Giordani S

(2021) Editorial: Photochromic

Materials: Design and Applications.

Front. Mater. 8:720172.

doi: 10.3389/fmats.2021.720172

\section{INTRODUCTION}

Materials able to respond to external stimuli have garnered interest in a variety of research areas. Ordinarily referred to as "smart materials", these sophisticated structures are capable of distinguishing and adjusting their responses to different triggers (Schnurbus et al., 2020; Qian et al., 2021). In this context, the chromic materials have been considerably focused for designing devices for multiple applications. Photochromism can be described as a reversible conversion of a chemical species between two isomeric forms caused by electromagnetic radiation absorption, resulting in a color change (Reis et al., 2020). Figure 1A shows the evolution of publications over the years considering the keyword photochromism on the Scopus website. Based on the recorded data, Figure 1B shows that the two areas with the highest number of publications include chemistry and materials science.

Light is one of the most attractive external stimuli to promote changes in chromic materials, since it is easy to control and non-destructive with high spatial and temporal precision (Li et al., 2020). In addition to color changing, some properties of the photochromic molecules and their materials may be tuned by light irradiation, such as electronic spectra, electrochemical properties, dipole interactions, and capability to coordinate. Photochromism can be observed in both organic and inorganic compounds, as well as their hybrid materials. This responsive behavior to light irradiation can be found in some metal oxides, alkaline earth, metal halides, and some transition metals (Prado et al., 2011). The promising organic classes of photochromic molecules include dithienylethene, spiropyran, and azobenzene (Zhang et al., 2013). Among these different classes of photochromic materials, the organic and its hybrid organic-inorganic types have been shown to be fatigue resistant with structural diversities. Applications of these materials include security markings, ions and solvents probing, optical shutters, photo-switchable molecular devices, topographical change materials, and optical memory storage systems (Klajn, 2014).

In this Research Topic, great examples of photochromic materials are described.Raymo et al. described the synthesis and spectroscopic characterization of four molecular switches with ratiometric fluorescence response in their paper entitled Switchable Coumarins for Ratiometric $p H$ Sensing. Based on their data, the equilibria between closed and open rings are achieved by trifluoroacetic acid increments in dyes solutions. These results from electronic spectra are a consequence of the structural transformation of the carbon atom at the junction of the indole and either the oxazine or the oxazolidine heterocycle, increasing the extended electronic delocalization. The emission spectra data for all molecules reveals an intense band between 454 and $480 \mathrm{~nm}$ for the closed ring forms, however, upon acidification this emission band decreases in intensity with the concomitant appearance and growth of a new band at $647-666 \mathrm{~nm}$. The chemical equilibria 


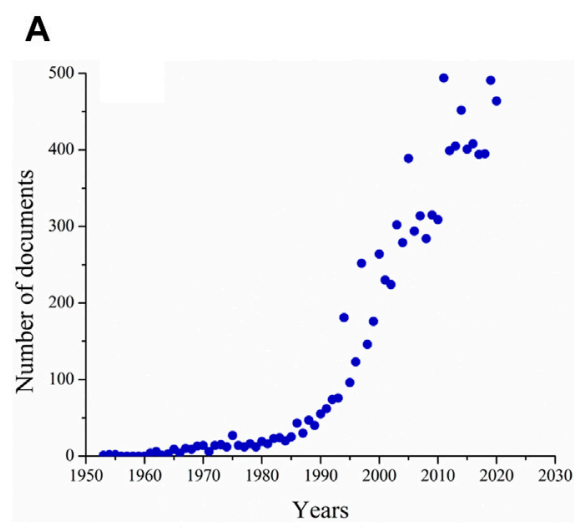

\section{B}

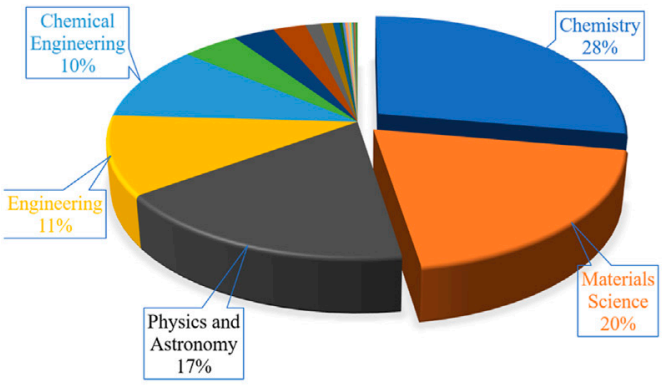

Chemistry

Materials Science

Physics and Astronomy

Engineering

Chemical Engineering

Biochemistry, Genetics and Molecular Biology

Computer Science

Mathematics

Pharmacology, Toxicology and Pharmaceutics

Pharmacolo

Environmental Science

Medicine

Agricultural and Biological Sciences

Multidisciplinary

Social Sciences

Business, Management and Accounting

Earth and Planetary Sciences

Health Professions

Immunology and Microbiology

Arts and Humanities

Psychology

Economics, Econometrics and Finance

Uconomics,

FIGURE 1 | (A) Number of publications per year and (B) distribution areas of publications based on the Scopus database records.

between these two different species with their relative emission intensities changing based on the $\mathrm{pH}$ can allow for independent detection. Although the closed forms are hydrophobic, they can obtain photophysical properties by adding a surfactant molecule dye.

In another paper, authors have combined knowledge from different areas such as synthetic materials, materials engineering, analytical chemistry, optics, electronics, and programming. McCaul et al. reported a multichannel fluidic platform in which the flow behavior can be controlled using light through the incorporation of photo-responsive soft-gel actuators based on spiropyran molecules in their paper entitled Fluidic Platforms Incorporating Photo-Responsive Soft-Polymers Based on Spiropyran: From Green Synthesis to Precision Flow Control. In this sense, a new optimized synthetic method for the synthesis of spiropyran in Gram quantities was proposed, as well as its chemical bonding to the hydrogel. After designing a fluidic channel in the swollen gel, the photoswitch to contract the gel exists when the protonated merocyanine form (colored yellow isomer) changes to the spiropyran (SP) isomer after light irradiation (UV at $450 \mathrm{~nm}$ ) irradiation. When the LED is turned off, SP spontaneously reverts to merocyanine and the valve closes.

In addition, Bertarelli et al. studied the topic in their entitled paper Molecular Design of Amphiphilic Plasma MembraneTargeted Azobenzenes for Nongenetic Optical Stimulation. These six different structures, sharing the same azobenzene core, were investigated by means of their affinity for the membrane environment, their isomerization ability, and electrophysiological effects using absorption/photoluminescence spectroscopy and confocal microscopy. The in vitro experiments evidenced the molecule's preference for the membrane environment rather than for their initial aggregate state observed in the aqueous vehicle solvent. In addition, photoreaction time constants of the aze-ammonium- terminated compounds are faster than the other ones. These findings can help to develop new candidate molecules for biosensors, biophotonics, biomedicine, and opto-neuroscience.

Lastly, Ribeiro et al. designed nanocomposites obtained by soaking phosphotungstic acid in wet bacterial nanocellulose membranes in their paper entitled Self-Supported Smart
Bacterial Nanocellulose-Phosphotungstic Acid Nanocomposites for Photochromic Applications. They combined organic-inorganic compounds based on bacterial nanocellulose and phosphotungstic acid to produce paper-like photochromic materials. These paper-like materials demonstrated reversible photochromic behavior based on the equilibrium between different tungsten oxidation states. The color change from colorless to blue is achieved by UV light irradiation with the blue color being UV dose-dependent. The mechanism described is related to the reduction of $\mathrm{W} 6+$ to $\mathrm{W} 5+$ and organic matrix oxidation, however, if the membranes are kept under nitrogen atmosphere or vacuum the blue color of the membrane remains longer than 45 days. These composite materials can be used in sensitive displays, smart windows, and other devices dependent on stimuli-responsive systems.

Currently, a variety of photochromic materials have been designed due to the evolution of chemistry and materials science strategies. Additional areas such as physics, biology, and nanotechnology have a lot to contribute to future photochromic materials. In addition, smart photoresponsive devices with singular functions have emerged as new opportunities for optic/electronic devices, drug delivery systems, environmental science, sensors, and so on. However, several challenges remain for future practical applications of photochromic materials including the ON/OFF ratio, response time, and the maintenance of photochromism on surface materials.

\section{AUTHOR CONTRIBUTIONS}

All authors listed have made a substantial, direct, and intellectual contribution to the work and approved it for publication.

\section{ACKNOWLEDGMENTS}

Host editors sincerely thank all authors, reviewers, and editors who supported this Research Topic and Frontiers in Materials for allowing us to edit it. We also thank all editorial staff who assisted us in this journey. 


\section{REFERENCES}

F Reis, I., Miguez, F. B., Vargas, C. A. A., Menzonatto, T. G., Silva, I. M. S., VeranoBraga, T., et al. (2020). Structural and Electronic Characterization of a Photoresponsive Lanthanum(III) Complex Incorporated into Electrospun Fibers for Phosphate Ester Catalysis. ACS Appl. Mater. Inter. 12, 28607-28615.doi:10.1021/acsami.0c03571

Klajn, R. (2014). Spiropyran-based Dynamic Materials. Chem. Soc. Rev. 43, 148-184.doi:10.1039/c3cs60181a

Li, C., Iscen, A., Palmer, L. C., Schatz, G. C., and Stupp, S. I. (2020). Light-Driven Expansion of Spiropyran Hydrogels. J. Am. Chem. Soc. 142, 8447-8453.doi:10.1021/jacs.0c02201

Prado, R., Zayat, M., and Levy, D. (2011). Photochromic Organic-Inorganic Hybrid Materials. Chem. Soc. Rev. 40, 672-687.

Qian, H., Purwanto, N. S., Ivanoff, D. G., Halmes, A. J., Sottos, N. R., and Moore, J. S., (2021). Fast, Reversible Mechanochromism of Regioisomeric Oxazine Mechanophores: Developing In Situ Responsive Force Probes for Polymeric Materials. Chem 7, 1080-1091.doi:10.1016/j.chempr.2021.02.014

Schnurbus, M., Kabat, M., Jarek, E., Krzan, M., Warszynski, P., and Braunschweig, B. (2020). Spiropyran Sulfonates for Photo- and pH-Responsive Air-Water Interfaces and Aqueous Foam. Langmuir 36, 6871-6879.doi:10.1021/acs.langmuir.9b03387
Zhang, J., Zou, Q., and Tian, H. (2013). Photochromic Materials: More Than Meets the Eye. Adv. Mater. 25, 378-399.doi:10.1002/ adma.201201521

Conflict of Interest: The authors declare that the research was conducted in the absence of any commercial or financial relationships that could be construed as a potential conflict of interest.

Publisher's Note: All claims expressed in this article are solely those of the authors and do not necessarily represent those of their affiliated organizations, or those of the publisher, the editors and the reviewers. Any product that may be evaluated in this article, or claim that may be made by its manufacturer, is not guaranteed or endorsed by the publisher.

Copyright (๑) 2021 De Sousa, Alexis and Giordani. This is an open-access article distributed under the terms of the Creative Commons Attribution License (CC BY). The use, distribution or reproduction in other forums is permitted, provided the original author(s) and the copyright owner(s) are credited and that the original publication in this journal is cited, in accordance with accepted academic practice. No use, distribution or reproduction is permitted which does not comply with these terms. 